\title{
Estrategia de implantación de un clúster de turismo en Nahuizalco, Sonsonate, El Salvador
}

\author{
Blanca Ruth Gálvez Rivas ${ }^{1}$ \\ Rosa Patricia Vásquez de Alfaro² \\ Óscar Armando Melgar Nájera ${ }^{3}$ \\ Recibido: 13/08/2013 - Aceptado: 02/09/2013
}

\section{Resumen}

Los clúster son una forma de exponer la ventaja competitiva por medio de la localización y cómo las comunidades o regiones alcanzan niveles de crecimiento y desarrollo económico. Una de las estrategias que se crea para incorporar dentro de la implantación del clúster es la ruta interna turística que incluye las cuatro riquezas principales que posee el municipio de Nahuizalco, identificadas en investigaciones que anteceden esta propuesta. Existen algunas limitaciones, como intereses a nivel individual en los sectores que conforman el municipio. Para aprovechar la riqueza que posee el lugar es necesario trabajar la asociatividad con los sectores involucrados; sin el desarrollo de esa variable no se podrá desarrollar ninguna propuesta de ruta turística u otro proyecto que se relacione con el turismo. Existen grupos celosos con su cultura y costumbres que se vuelven obstáculo en el progreso local. Con la validación de los resultados, se propone el diseño de la ruta turística interna que contempla la riqueza del lugar. Esto es atractivo a los ojos de cualquier turista nacional o internacional, así como para cualquier empresa que se dedica a la comercialización de paquetes turísticos, contribuyendo al desarrollo económico del municipio.

\section{Palabras clave}

Desarrollo. desarrollo local, turismo-El Salvador, desarrollo de la comunidad, comercio turístico, promoción de turismo.

\begin{abstract}
Clusters represent a form of competitive advantage where location is a main attribute for communities and regions to achieve levels of economic growth and development. One of the strategies created to incorporate within the implementation of the cluster is a domestic tourist route which includes four main riches or attractions for Nahuizalco township identified in investigations preceding this one. There are some limitations as some sectors of the municipality show individual-level interests that prevent to take advantage of the wealth diversity they own. It is necessary to work the concept of association with stakeholders, for them to take advantage of the tourist route based on the community riches. The groups' jealousy of their customs and traditions may become an obstacle for their community development.
\end{abstract}

With the validation of the results we propose the design of a domestic tourist route that includes the wealth of the place, this is appealing to the eyes of any national or international tourists as well as for any company dedicated to the commercialization of tourist packages, contributing to the economic development of the municipality.

\section{Keywords}

Development, local development, tourism - El Salvador community development, tourism trade, tourism promotion.

1 Blanca Ruth Gálvez Rivas. Máster en Docencia Universitaria, máster en Metodologías de Investigación, licenciada en Administración de Empresas, docente investigadora de la Facultad de Ciencias Empresariales. Universidad Tecnológica de El Salvador. bruth@utec.edu.sv

2 Rosa Patricia Vásquez de Alfaro. Máster en Docencia Universitaria, licenciada en Administración de Empresas, docente investigadora de la Facultad de Ciencias Empresariales. Universidad Tecnológica de El Salvador. pvazquez@utec.edu.sv

3 Óscar Armando Melgar Nájera Máster en Educación Universitaria, licenciado en Contaduría Pública, docente investigador de la Facultad de Ciencias Empresariales. Universidad Tecnológica de El Salvador. omelgar@utec.edu.sv 


\section{Introducción}

El turismo tiene un impacto cada día más cercano con la realidad del lugar visitado, y El Salvador no es la excepción, por lo que, en los últimos años, se visualizan muchas rutas turísticas con atractivos que poseen sus pueblos, territorios, su historia, con acontecimientos que para las nuevas generaciones es de mucho valor. Es así como el turista quiere conocer detalladamente los lugares en viajes cortos y frecuentemente a costos bajos. Al ofrecer rutas turísticas internas de rutas que son más globales se abre una mayor oferta a aquellos que están encargados de ofrecer estos productos y benefician a las localidades que participan. El municipio de Nahuizalco, al ofrecer esta ruta, se acopla a las necesidades del nuevo usuario porque permite hacer varios tipos de turismo a la vez, como son el cultural, natural, arquitectónico y artesanal.

La ruta turística estimula la cooperación y la relación directa entre las diferentes empresas de los municipios o regiones que conllevan aun desarrollo de clúster (Lopez, 2008). Consultado en http://mdc.ulpgc.es/cdm/ref/collection/pasos/ id/305\#img_view_container el 14 de agosto, 2012.

Un ejemplo en nuestro país es la Ruta de las Flores. Por tanto, el turismo y la creación de rutas es una alternativa para llevar a cabo estrategias de desarrollo y restablecimiento económico y social de áreas rurales, siendo la asociación una de las formas organizativas con más futuro en el campo del turismo debido a que se puede adaptar de una manera más rápida a las exigencias de la demanda (Fernández y Puig, 2002).

Esta investigación comprende el levantamiento del inventario turístico del municipio para diseñar el recorrido turístico interno que concatene las cuatro riquezas que posee el municipio (arquitectónica, artesanal, cultural y natural), consolidando una oferta turística atractiva para el visitante como una estrategia de desarrollo en el área turística. Partiendo de la validación de los resultados que se plantearon en el estudio de "Conformación de clúster de turismo en el municipio de Nahuizalco" (Galvez, Vásquez \& Melgar, 2011), se conformarán las actividades y sectores de apoyo que participaran en la implantación, fomentando la actividad turística y con ello lograr un desarrollo económico del municipio.

\section{Rutas turísticas}

Para ubicarse en este contexto se consideraron algunos elementos básicos para conformar una ruta turística, como:
Establecimiento de un eje temático o hecho diferencial claramente identificable por el público objetivo.

- Catalogación de puntos y elementos de mayor atractivo a partir de los recursos identificados en el inventario.

Dosificación de los atractivos, alternando puntos de mayor y menor interés.

\section{Tipos de rutas}

Se hace un desglose de las diferentes rutas que se conocen en el ámbito turístico.

\section{Turismo cultural}

La riqueza histórica que se atesora en el seno de muchas familias criollas constituye, si no el único, uno de los principales sustentos de propuestas de turismo rural basadas en la cultura.

Existen numerosos establecimientos agropecuarios que poseen un patrimonio histórico muy valioso, que solo puede ser conservado gracias a la explotación turística.

\section{Turismo de aventura}

Utiliza el entorno o medio natural como recurso para producir sensaciones de descubrimiento, por lo que requiere consecuentemente de espacios con poca carga turística y, mejor aún, poco explorados. Las actividades que se realizan son muy diversas y dependen del entorno natural en el que se sitúe el predio. Así un río, por ejemplo, genera condiciones propicias para el rafting o canotaje; la montaña, para el andinismo, además de otras actividades que pueden realizarse en casi todos los ambientes, como cabalgatas y senderismo.

\section{Rutas gastronómicas}

Según Barrera (1999), una ruta gastronómica es un itinerario que permite reconocer y disfrutar de forma organizada el proceso productivo agropecuario, industrial y la degustación de la cocina regional; a su vez, las rutas gastronómicas se organizan en torno a un producto clave que caracteriza la ruta y le otorga su nombre. La ruta debe ofrecer a quienes la recorren una serie de placeres y actividades relacionadas con sus elementos distintivos: comida, producción agroindustrial; actividades rurales, entretenimientos en la naturaleza y actividades propias de la cultura regional.

\section{Rutas temáticas e interpretativas}

Ríos (2006) la define como recorridos que conectan puntos relacionados, ya sea de carácter histórico, social o artístico. Generalmente las rutas tienen algún tipo de señalización, 
y básicamente están pensadas para realizarse a pie. En cuanto al equipamiento está destinado al público en general; su emplazamiento está generalmente vinculado a otros tipos de servicios, tales como centros de visitantes, zonas recreativas, áreas de camping, entre otros.

\section{Método}

La investigación se enmarca en la modalidad de investigación-acción. Se pretende, a partir de los resultados, obtener una propuesta y/o estrategia de implantación de una de las líneas que se plantearon en el estudio de Conformación de Clúster de Turismo en el municipio de Nahuizalco (Gálvez, Vasquez \& Melgar, 2011), por lo que se optó por un diseño metodológico que permitiera aplicar la propuesta que se hiciera en ese estudio.

El diseño metodológico que se aplicó para desarrollar la propuesta es la validación de resultados del referido estudio en el 2011. Se trabajó la viabilidad de desarrollar una ruta turística interna en el municipio de Nahuizalco, por lo que se identificó el inventario turístico que posee el lugar en sus cuatro áreas: cultural, arquitectónica, natural y artesanal que conforman la ruta interna. El desarrollo de la investigación enmarcó los elementos: participantes: personas mayores de edad, de entre 18 y 65 años, en ambos géneros, con ocupaciones empresariales; y población en general en condiciones de salud aceptables. Se utilizó el muestreo dirigido, seleccionando personas que proporcionen información adecuada y necesaria para el estudio. Los instrumentos para recolectar la información fueron: guía de entrevista dirigida, guía de observación y cuestionario, los cuales fueron revisados por un experto en diseño de rutas turísticas. Se le presentó el diseño del cuestionario para obtener la información de la viabilidad del desarrollo de la ruta, el cual fue autorizado después de hacer algunos ajustes en el diseño.

La guía de entrevista dirigida, como medio de validación cualitativa de la investigación, valoró las aportaciones que los diferentes actores brindaron al participar en el focus group.

La guía de observación se utilizó para documentar visualmente todos los lugares visitados en el levantamiento del inventario turístico del municipio de Nahuizalco, utilizando equipo especializado para obtener información que ayudó a documentar aspectos relacionados con el estudio; entre lo más sobresaliente, lo relacionado con la naturaleza, como las cascadas, los jardines, los sembradíos de hortalizas y otros. En el perfil arquitectónico: la fuente, la iglesia, por citar algunos. En la línea de artesanías: Ios talleres, las exposiciones, los negocios, etc. En la línea cultural: las cofradías, bailes, fiestas patronales, etc.
El cuestionario se administró a una población aleatoria del casco urbano del municipio. Se encuestaron personas que desarrollan actividades relacionadas con el turismo, mayores de edad de ambos sexos, lugareños que tengan su negocio relacionado con el turismo y otros que indirectamente contribuyan al fortalecimiento de este.

El procedimiento aplicado en el estudio es parte de la aplicación de una de las conclusiones realizadas y planteada en la investigación realizada en el año 2011 (Gálvez, Vásquez \& Melgar, 2011) sobre la estructura de un clúster de turismo en el municipio de Nahuizalco.

El equipo investigador elaboró los instrumentos y participó en el trabajo de campo, así como en la dirección del focus group y las guías de observación y su aplicación.

\section{Resultados}

El municipio de Nahuizalco cuenta con un potencial turístico caracterizado en cuatro riquezas (natural, arquitectónica, cultural y artesanal), que se convierten en el atractivo turístico del lugar, entre los cuales se encontraron las cascadas, los senderos de siembra de hortalizas, balnearios, ríos, la fuente, las iglesias, jardines, entre otros, que no se explotan por falta de coordinación de los sectores de apoyo y conexos y los sectores directamente relacionados que conforman ese lugar.

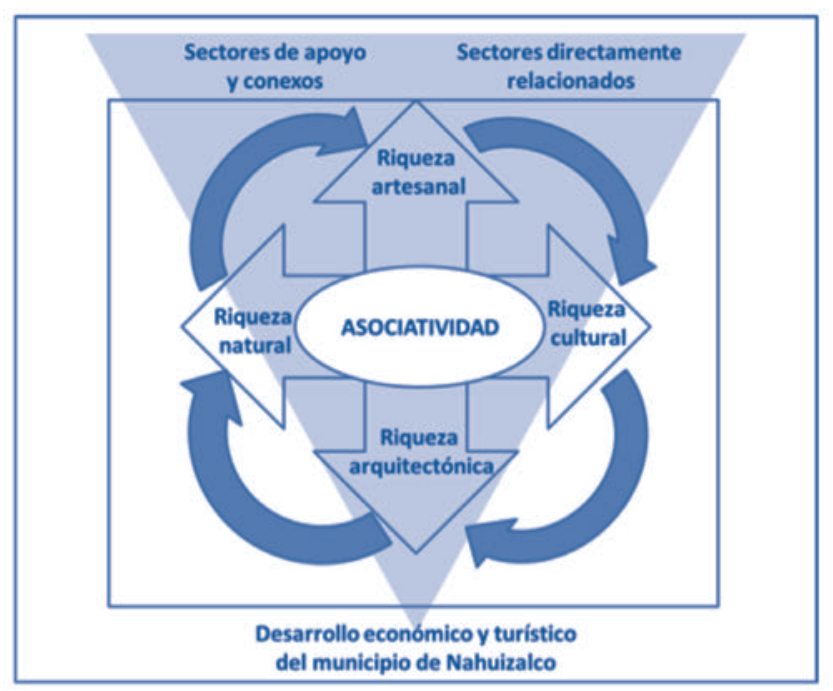

Figura 1.

Figura 1. Desarrollo económico y turístico del municipio de Nahuizalco, departamento de Sonsonate. Elaboración propia, 2012; una forma de integrar los elementos importantes de los resultados del estudio. 
Según los resultados, se determina que hay diferentes formas de desarrollar un lugar; pero que en este caso, se debe trabajar orientado a la búsqueda de la asociatividad para hegemonizar las cuatro riquezas que se observan en el municipio, a fin de desarrollar actividades conjuntas encaminadas a mejorar la economía del municipio.

Se identifican diversos grupos de indígenas que protegen su identidad cultural, costumbres y tradiciones, aspectos que hacen proteger su territorio, que luego pasan a ser un fuerte obstáculo para desarrollar la parte turística y, por ende, el desarrollo económico, razón por la cual la actividad turística en Nahuizalco no es tan dinámica como en otros municipios que tienen estas mismas características.

La asociatividad se vuelve un elemento fundamental en el desarrollo integrador de la riquezas (natural, arquitectónica, cultural y artesanal) y de la identidad cultural, teniendo como respuesta el diseño y la implantación de la ruta turística interna en donde se consideren los sectores de apoyo conexos planteados y los sectores directamente relacionados que permitan, paso a paso, la conformación del clúster de turismo propuesto. Cuando se logre integrar todos estos elementos en un asocio entre ellos, se podrá ver un desarrollo económico y turístico en el municipio.

Dentro de las actividades que se deben desarrollar para poder lograr el objetivo de crear una ruta turística de una manera sostenible y que se convierta en un interés turístico nacional para impulsar Nahuizalco y crear condiciones desarrollo económico local, se deben seguir los siguientes pasos: asociatividad, infraestructura turística, seguridad, oferta turística y diseño de rutas turísticas.

\section{Discusión}

Las rutas turísticas tienen un gran potencial en cualquier lugar del mundo, y no sería la excepción en El Salvador; pero deben crearse las condiciones adecuadas para que estas se desarrollen.

El municipio de Nahuizalco posee las características potenciales para desarrollarse rutas turísticas, como el turismo rural, cultural, de aventura, gastronómico, etc.; una ruta turística interna que integre estos aspectos y conlleve el desarrollo en esta área, aunque se deben crear las condiciones mínimas para que esta se implante. Se considera que se debe iniciar un proceso de emprendimiento para crear ofertas turísticas reales y que el actor principal sea la comunidad.
Por otra parte, se debe trabajar en la orientación de asociaciones que integren los representantes de los diferentes sectores que velan por la protección de su cultura y sus costumbres en dicho lugar. Al crearse la propuesta de la ruta turística debe legalizarse el recorrido, y estos actores deben solicitar permisos pertinentes a los propietarios para poder utilizar los senderos donde se propone que pase la ruta de agroturismo y turismo de aventura, para no generar problemas a los propietarios de las parcelas. Además, habrá que involucrar otros actores como inversionistas, tanto internos como externos, para ofrecer al turista un buen servicio y que se sienta acogido por el municipio.

La propuesta que se presenta integra las cuatro riquezas del municipio para explotarlas en sus diferentes aspectos y que el turista conozca las maravillas que posee el lugar. Dentro de las propuestas están:

1. Ruta casco urbano (cultural, arquitectónico y artesanal). Esta ruta permite tener un encuentro con las manos laboriosas de Nahuizalco, su gente, sus costumbres y su entorno cultural y social. En este recorrido se podrá apreciar lo siguiente: artesanía, alimentos típicos, cultura, expresiones culturales y arquitectura.

\section{Ruta turismo rural (natural)}

Para el desarrollo de estos recorridos se propone realizar dos rutas. Estas son:

- Ruta de agroturismo

La caminata se realiza a través de las diferentes parcelas cultivadas que se puede visualizar en la zona del cantón Pushtán. Estos cultivos son: hortalizas, verduras, maíz, maicillo, tule, bambú, entre otros.

- Ruta de turismo de aventura La propuesta considera una caminata por los senderos hacia la cascadas Las Golondrineras, ubicadas en el cantón Pushtán, a $4 \mathrm{~km}$ del casco urbano de Nahuizalco; o la cascada el Salto, visitar la central hidroeléctrica, Sisimitepec, el jardín Tatalpa y luego volver al casco urbano.

Al desarrollar e implantar estas dos rutas se daría paulatinamente crecimiento a las áreas turística y económica y de mucho desarrollo en el municipio de Nahuizalco. 


\section{Conclusiones}

- Para Nahuizalco es una oportunidad de desarrollo económico y local al implantar la ruta interna; y debe considerarse una herramienta de desarrollo integral, con significado económico y contenido social; y así convertirse en el principal soporte de crecimiento económico por tratarse de un sector que favorece múltiples actividades relacionadas. Es necesario que el lugar ofrezca a los turistas las condiciones mínimas, como, por ejemplo, un hostal, restaurantes, transporte, comercio, casa de descanso, etc.

- El municipio debe integrar a los diferentes actores como: los indígenas, artesanos, productores, ganaderos y microempresarios, asociándolos; y que hegemonicen cada actividad y puedan beneficiarse en los ámbitos comunal y municipal, al implantar las rutas internas del municipio.

\section{Referencias}

Fernández M.M. y Puig M.A. (2002), “El papel del cooperativismo en el turismo rural de la Comunidad Valenciana", Ciriec-España, 41: 183-212.

Barrera E. (1999), "Las rutas gastronómicas, Una estrategia de desarrollo rural integrado". Consultado en: enhttp://www.agro.uba.ar/sites/default/files/ turismo/publicaciones/barrera/LAS_RUTAS_ GASTRONOMICAS_1999.pdf, el 18 de septiembre, 2012.

Gálvez R., Vasquez, Melgar N, (2011), "Conformación de Clúster de Turismo en el municipio de Nahuizalco, Sonsonate".

Ríos P., Rubilar L. (2006), Diseño y comercialización de la ruta de la ecoaventura, Ensenada, Chile. 